\title{
Integration of PC-based 3D Immersion Technology For Bio-mimetic Study of Human Interactive Robotics
}

\author{
Z.W. Luo ${ }^{1)}, \quad$ M. Onishi ${ }^{1)}, \quad$ T. Odashima ${ }^{1)}, \quad$ K. Oyama ${ }^{2)}, \quad$ F. Asano ${ }^{1)}, \quad$ S. Hosoe ${ }^{1,2)}$ \\ 1) Bio-Mimetic Control Research Center, RIKEN \\ 2) School of Engineering, Nagoya University \\ luo@bmc.riken.go.jp
}

\begin{abstract}
Two novel immersion type experimental platforms integrating low cost PC-based $3 D$ VR technology are developed and actively used for bio-mimetic study on new paradigm of human interactive robotics. The first platform integrated PC-based immersion-type display with a motion capture system as well as a 3D dynamic simulator, which makes it possible for human subject to feel as if he/she is immersed within the same environment of the robot. It can be used to simulate and evaluate the physical interaction with human while not really damage him/her. The second one consists of real and virtual dual-arm robot equipped with vision and force sensors. Through force display and HMD, human operator can feel as if he/she is immersed within the robot body and perform tele-manipulation easily based on our unilateral control approach.
\end{abstract}

\section{Introduction}

The next generation of robots is highly expected to interact with human directly and support our every day life. Towards this dream, it is necessary for us to develop new design, simulation, as well as human interactive evaluation technologies. There are many differences between industrial robots in the factory and the robot that will play an active part in a general environment. For example, although speed and accuracy of operation are important for the industrial robot, the robot in our home should be evaluated based on its adaptability and safety. Moreover, the human subjective evaluation of the robot's affinity such as its form, color and feeling are also important. Therefore, when considering the development and application of human interactive robots, human subjects should be included in a robot's design and experiment environment in a natural way.

Experiment of physical interaction between human and a robot with large force is very dangerous and has high risk. Although the approach to use a dummy doll instead of human being can be considered, the subjective evaluation of the robot cannot be obtained from the target human directly. Considering these facts, in this research, we construct two novel immersion type simulation environments for developing human interaction robot. The first system uses low cost AT-clone computers to calculate 3D dynamics and collision of a robot when it interacts with human and projects the audiovisual results using an immersion type display. Through dynamic calculation of the robot, human subject can feel as if he/she is together with the virtual robot in the same environment naturally, and can specify force on the robot directly in real time. This simulation environment makes it easer to design and evaluate the form and performance of a robot with high safety and sufficient accuracy.

Our second immersion platform consists of a real and a virtual dual-arm robot equipped with vision and force sensors. Through force display and HMD, human operator can feel as if he/she is within the robot body and perform tele-manipulation easily using our simple unilateral control approach [1].

In the following paper, we first describe the $3 \mathrm{D}$ immersion-type dynamic simulation environment and its applications in section 2. We then show our second platform and its application in unilateral tele-manipulation in section 3 followed by the conclusion in section 4 .

\section{Immersion-type 3D Dynamic Simulator}

\subsection{System Construction}

The first immersion type 3D dynamic simulation platform we constructed is shown in Fig.1. This system consists of three main parts:

(1) An immersion type display,

(2) A 3D dynamic simulator, and

(3) A motion capture part, respectively. Each part uses only two or more low cost PC computers instead of supercomputer as existing systems. All computers communicate with each other through LAN using TCP/IP.

The immersion type display part presents human with audiovisual information and tactile sensation, which makes it possible for the human subject to feel as if he/she is together with and can interact with the virtual robot directly.

The 3D dynamic simulator part calculates the motion of the virtual robot, which follows the given set of target angles while interacting with human subject. Here, both the gravity as well as the external force applied to the robot from human are considered in its dynamic calculation.

The target motion of the virtual robot is given based on the motion capture results of human motion. 


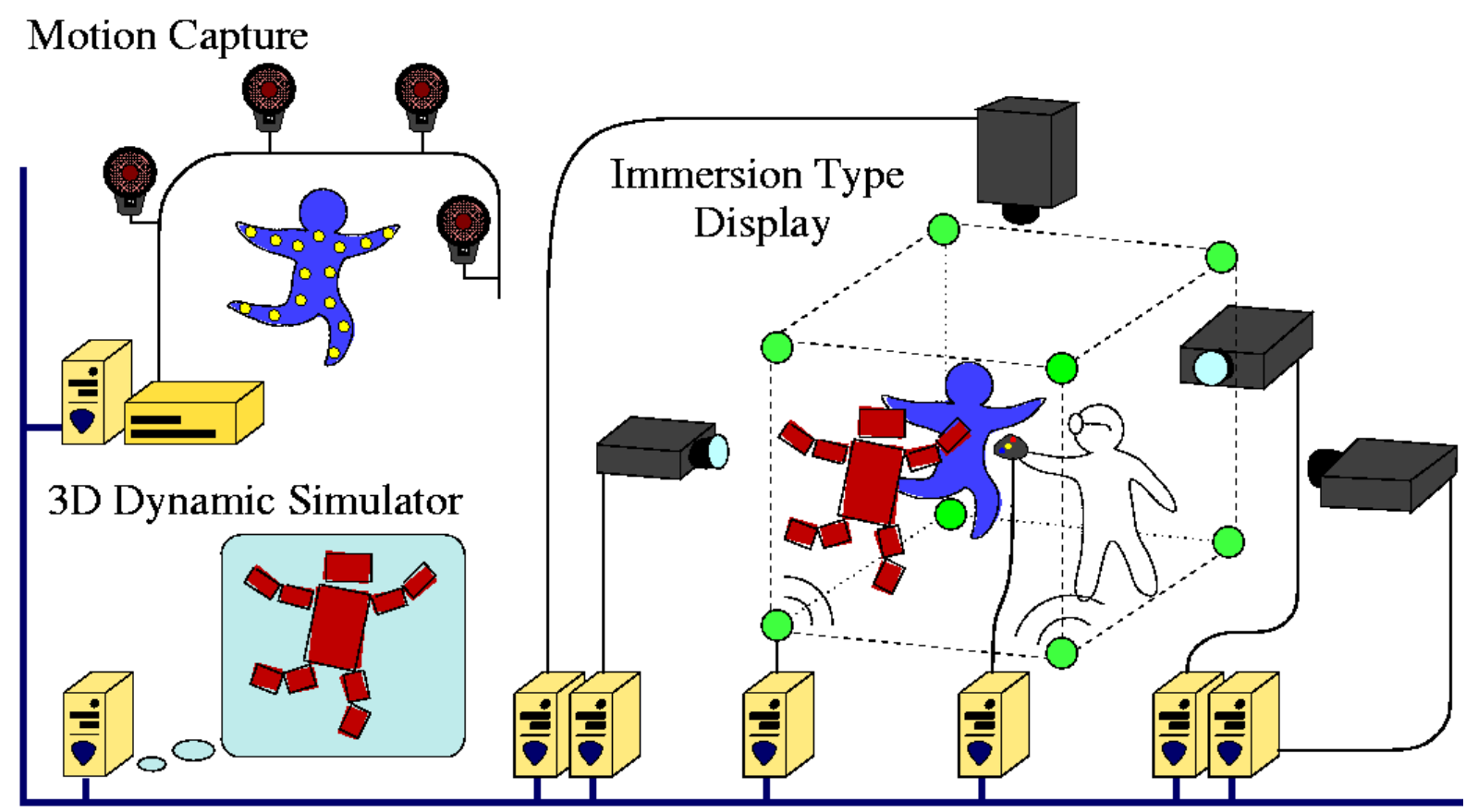

Fig.1 Setup of immersion type 3D dynamic simulation platform

\section{Dynamic Motion Capture Part}

Research of human interactive robot should be started from the measuring and analyzing of human dynamic motions.

Here, as shown in Fig.2, six high-speed infrared cameras is used to observe the markers attached on the human body with the sampling period of $120 \mathrm{~Hz}$. 8 force plates on the ground are set to measure the foot forces which will be used to calculate the human joint torques. Unlike other motion capture systems that differential the joint position data to solve joint acceleration within low frequency bandwidth, in our system, we use 32ches of EMG and $32 \mathrm{ches}$ of accelerometers to measure the joint acceleration directly, this together with the data of force plates can then be used to calculate the dynamic human motion more precisely. The measurement results are sent to the next two parts via TCP/IP for further processing.

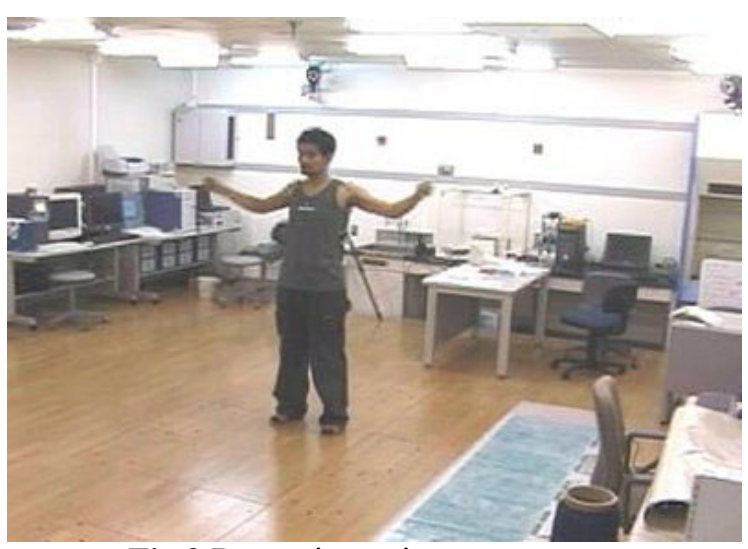

Fig.2 Dynamic motion capture part

\section{D Dynamic Simulator Part}

The 3D dynamic simulator as shown in Fig.3 calculates the position and orientation of the virtual robot. This dynamic simulator is coded by applying the programming library "Vortex" of CM-Labs Simulations Inc. Vortex has functions to process also the collision check between the links and the dynamic motion calculations of each link in real time. The calculated position and orientation of all links is transmitted to the immersion type display part using TCP/IP. The control torque, external force applied from the human subject and the gravity defined in the virtual space decide the motion of the virtual robot. Here, the external force from human subject is sensed using the input device named "WANDA" which is equipped in the immersion type display. The human subject can select to give the specific force to the specific part of the virtual robot.

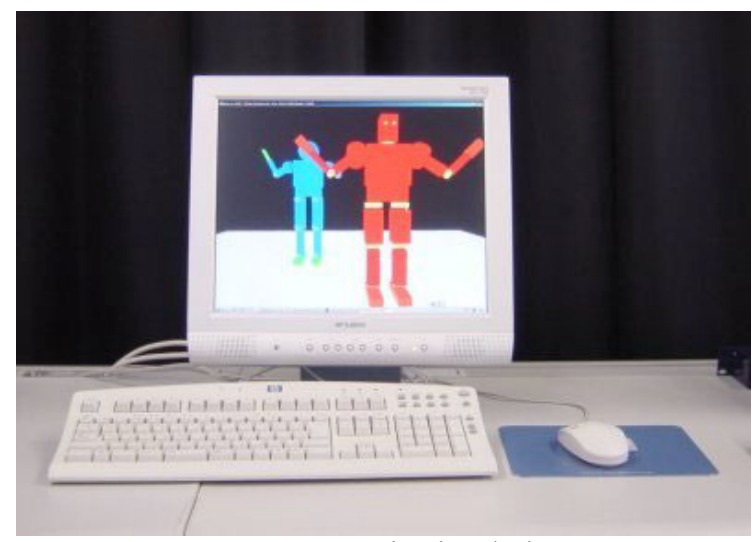

Fig.3 3DDynamic simulation part 


\section{Immersion type display part}

The central part of this system is the immersion type display. Here, as shown in Figs.4 and 5, unlike the use of high cost supercomputer, based on the parallel graphic processing of low cost $\mathrm{PC}$, the visual and auditory information presents the human subjects with high presence of the virtual robot.

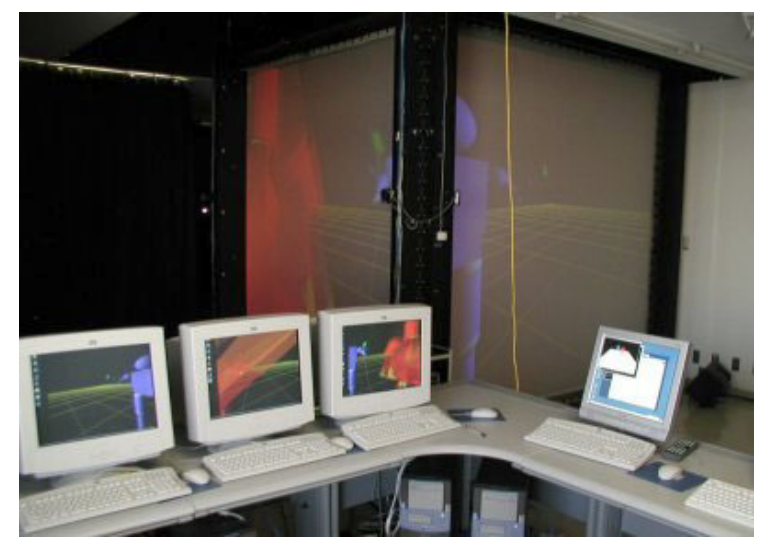

Fig.4 Parallel graphic processing based on PC

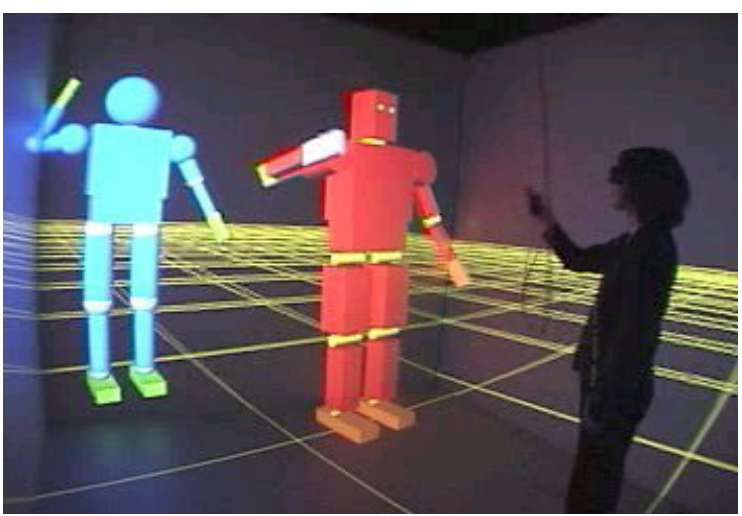

Fig.5 Human subject uses WANDA to pull the hand of the virtual robot within immersion type display

\section{(1) $3 D$ visual information}

The 3D visual information is projected by four 5000 ANSI DLP stereo projectors separately on four screens arranged as the cube surrounding the human subject. The high resolution of image and a large visual field angle provide a high reality feeling to the human subject.

\section{(2) $3 D$ auditory information}

3D auditory effect is realized using Huron of Lake Technology. 8 speakers are set at one corner of the immersion type display. This system can control two or more sound sources in real time and can generate the virtual ground sound space with high reality by the speakers array with respect to the robot motion, simultaneously.

\section{(3) WANDA and Position Sensors}

Within the immersion type display, human subject can use WANDA to control his/her view direction. In addition, two magnetic sensors are attached on the WANDA and the liquid crystal glass to measure both the head and the hand position of the subject.

\subsection{Applications}

As the application of this system, we studied perturbed human body motion control function that is, to study the response of human body by specifying an external force to him while he is performing some other motions. From the point of view of safety, we don't add force directly to human but rather use the virtual robot. In detail, we first measure the human motions and construct a virtual robot to replay the same motion via dynamic motion control. We then ask a human subject to interact with this virtual robot using WANDA within the immersion type display. The experiment results are shown in Figs.6 6 and 7.
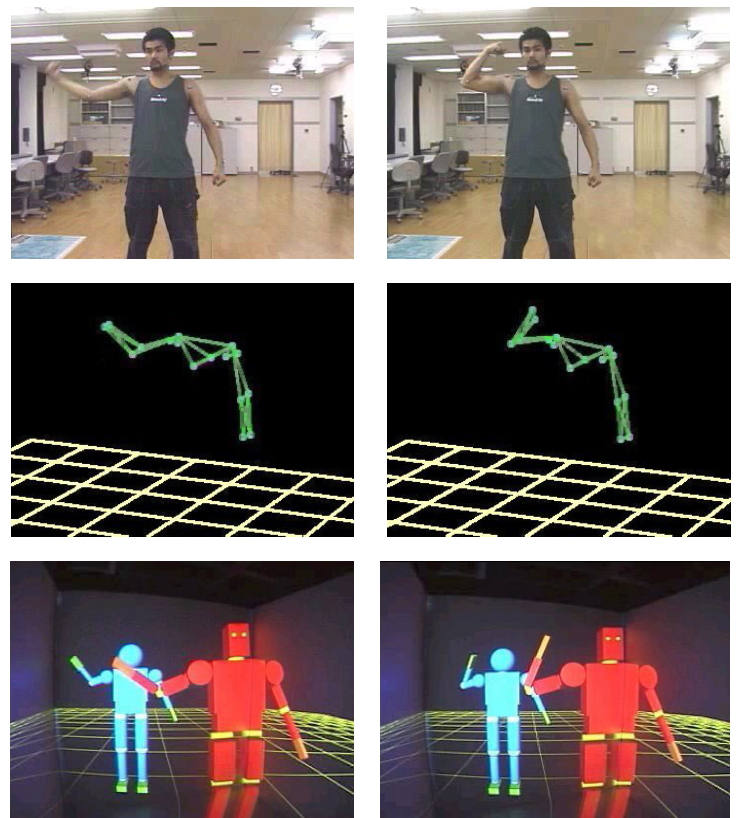

Fig.6 Dynamic control of a virtual robot to follow the same motion as human subject
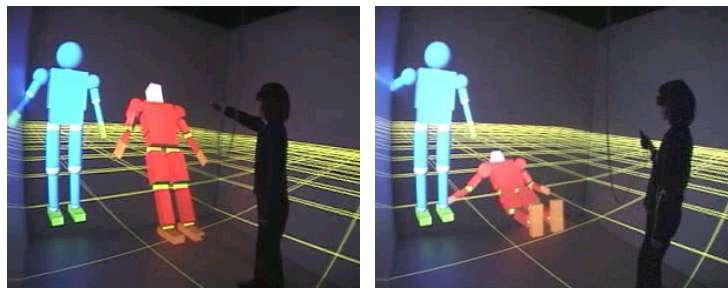

Fig.7 The human subject pushes the virtual robot

In Fig.6, the blue one is only a 3D CG, which always replays the measurement of human motions, while the red one is a virtual robot, which is dynamically controlled to follow the human motions. The perturbed result is showed is shown in Fig.7, where the human subject used WANDA to add an external force to the head of the virtual robot, which coursed the robot to turn down on the ground, and the crash sound can be heard from the exact position where the robot is crashed. Presently, it is impossible to react the force to the human subjects. It's left as our next research subject. 

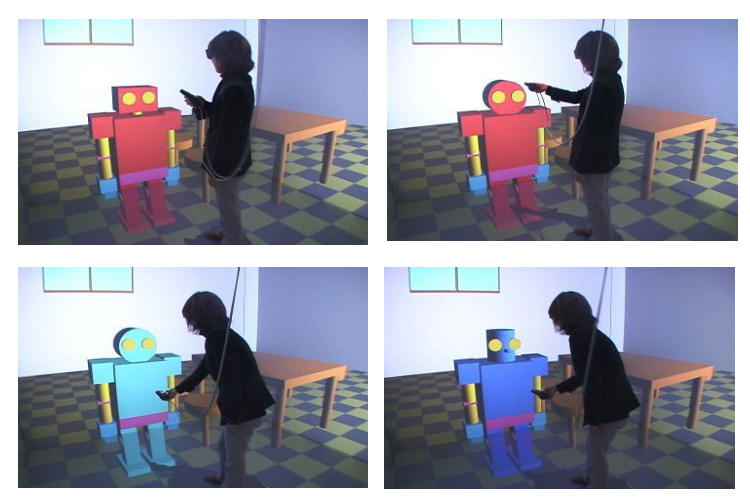

Fig.8 Human subject changes the design of the robot

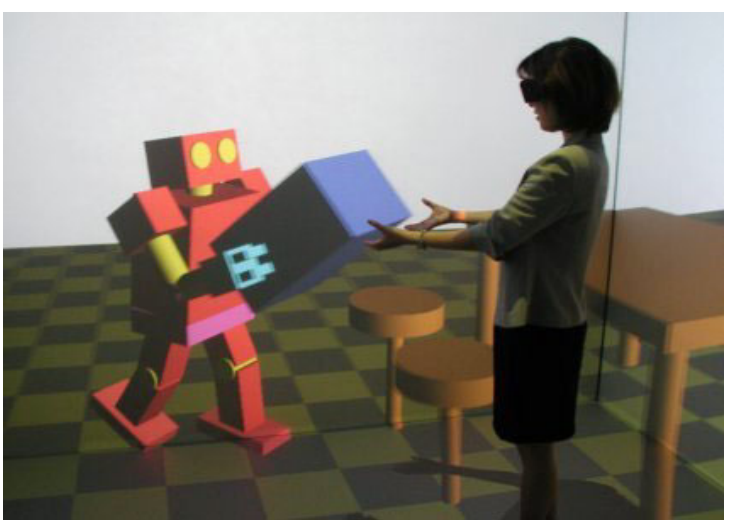

(a) Virtual robot sends a box to human subject
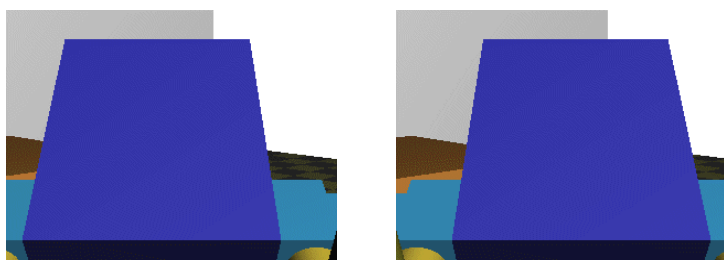

(b) Visual images as seen from virtual robot
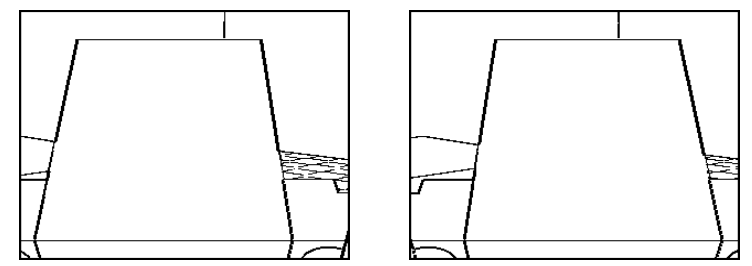

(c) Edgy detection from virtual visual images

Fig.9 The virtual robot can see its outside objects

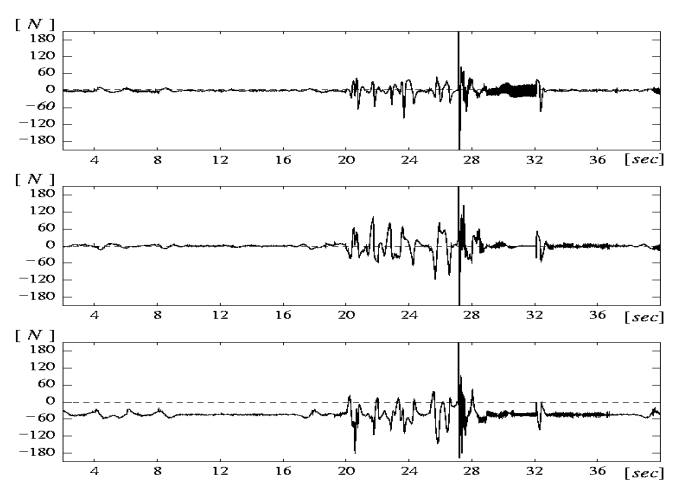

Fig.10 Force response from virtual force sensor
Using this system, it is easy for us to adjust the virtual robot's form, color and size. One example is shown in Fig.8. Here, the human subject uses the WANDA to select her favorite design.

In addition, the virtual robot also has its feeling via virtual eyes and virtual force sensor. For the task shown in Fig.9(a), the outside objects as seen from the robot is shown in Fig.9(b). The image processing part of the virtual robot can quickly calculate the object's edgy as shown in Fig.9(c) so as to recognize it and perform the cognitive movement. Fig.10 shows the force responses from the virtual force sensor, it can be found that there is a large impulse force generated when the robot crashed to the ground at about 27[s]. By using mixed reality technology, it will be expect that the robot can also see the real objects including human subject in the near future.

In summary, our system realized five original features:

(1) It is the first for us in Japan to realize all measurement, calculation and $3 \mathrm{D}$ multi-screens $\mathrm{CG}$ display using only low cost PC network.

(2) It displays 3D CG based on the dynamic calculations.

(3) It has high precision in dynamic analyzing of the human motions by directly using accelerometers.

(4) It is bright by using 5000ANSI DLP projector.

(5) The virtual robot not only has its dynamic body, but also has virtual vision and force sensing functions.

With this immersion type simulation system, it's possible for us to feel naturally as if we are sharing the common environment with the virtual robot, which makes it easer and more direct to design, test and evaluate of the next generation of human interactive robots.

\section{Immersion-type Tele-manipulation}

Unlike above immersion type system, which realized the effect such that the human subject can be immersed within the same environment together with the virtual robot, in this section, we construct another immersion type system, which can realize the effect such that the human operator can feel as if he/she is directly within the body of the virtual/or real robot. This technology can greatly expend our task ability as well as performance in the application of tele-manipulation.

\subsection{System Construction}

As shown in Fig.11, this system mainly consists of three parts, they are:

(1) A dual arm robot part with a stereovision head.

(2) A virtual robot part that has the same structure as the real one.

(3) The human/computer interface part including force and visual displays, respectively. 


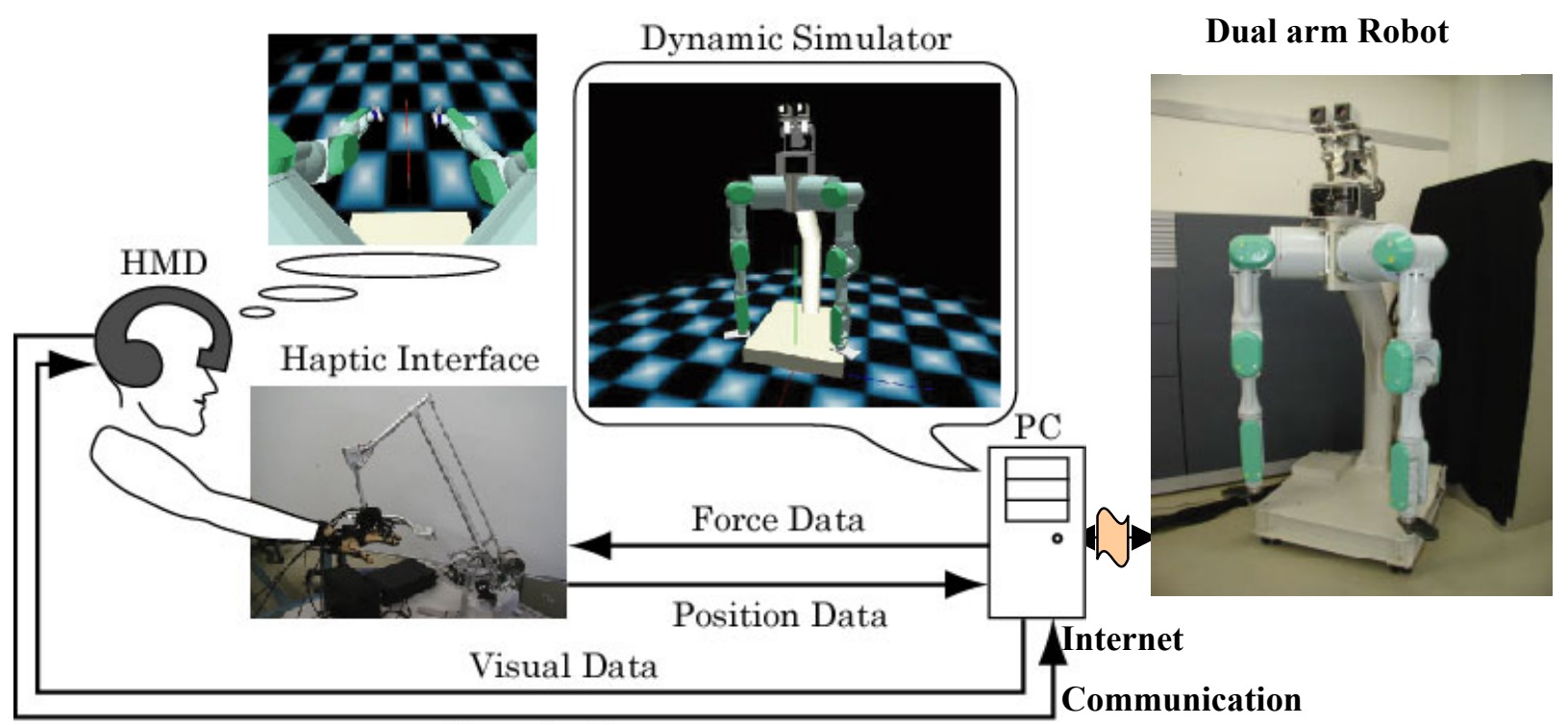

Rotation Data

Fig.11 Setup of immersion type tele-manipulation platform

\section{(1) Real robot part}

Each arm of the real robot part has 7 D.O.F of rotational joints, which is almost the same as the structure of human arm. A 3 D.O.F stereovision head is attached at the robot and has sound recognition/communication function. A 6 D.O.F. force sensor is attached at the end-effecter of the robot arm. The position, interaction force as well as vision information of the real robot are sent via internet to the PC of dynamic simulation part.

\section{(2) 3D Dynamic simulation part}

The PC of 3D dynamic simulation part constructs the same structure of a virtual robot as the real one. By applying the programming library "Vortex" of CM-Labs Simulations Inc, the simulator receives information from the human operator and the real robot and performs dynamic calculation to realize the dynamic motion. The simulator also has its virtual stereovision head and force sensors, which measure the virtual environment and send the information to human operator via interface part. The library "Vortex" basically supplies us three key functions:

a. Modeling and numerical calculation of dynamic equations of mechanical systems,

b. Collision detection of rigid objects, and

c. Rendering of 3D computer graphics, respectively.

\section{(3) Human interface part}

The human operator is equipped with a head mounted display (HMD) and two haptic arms with high resolution. The HMD displays the $3 \mathrm{D}$ visual images of the virtual/or real robot head and sends 3 D.O.F head rotation angles to control the positions of both the virtual and real robot head. Therefore, the operator can see where he/she wants to see. On the other hand, the haptic arm consists of:

a. A 6 D.O.F arm, which can measure the hand configuration and display 3 D.O.F of force vector at the operator's hand,

b. A glove that can measure 22 D.O.F. of the operator's finger configuration, and

c. A grasp that uses 5 wire-driven actuators to display 5 pushing forces between the robot (virtual or real) and its environment to the human operator so that the operator feels as if he/she is directly in contact with the same environment. respectively.

\subsection{Applications}

This simulator has three levels of applications, they are:

(1). The virtual robot along can be used easily to test various bio-mimetic control algorithms, such as learning cognitive behaviors.

(2). The simulator together with human interface can be used for training the human operator to perform some cooperative tasks.

(3). The overall system can be used to examine the cooperative tele-manipulation including the physical environmental interactions.

In the following, we mainly focus our research on its application in tele-manipulation. Concerned with this application, three critical problems should be discussed.

Firstly, although using dual arms as human can largely expend our task ability, it arises a serious problem of collision between each arm, which makes it difficult for the human to operate. In order to avoid the dangerous collision, it's necessary for the simulator itself to generate in real time the collision avoidance trajectories for each arm based on the position information from human operator. 
Generally, as seen from one arm, the other arm is always moving and is a very complex obstacle. By now, there existing no effective real time algorithm to solve such a complex dynamic obstacle avoidance trajectory generation task.

Thanks the basic collision detection function of the library "Vortex", in our system we simply solved this problem in the following way. That is, although we calculate and rendering the motion of the virtual dual arm based on the real size, shape and dynamics of the real robot, we perform collision check by considering the expended shape of the robot using collision detection function of the library. In the case when collisions are detected, the simulator will send back forces to the operator so as to prevent his/her further motion from real collision. As a result, human operators need not to worry about the arm collision problem and can perform operation easer.

The second problem in tele-manipulation is wither it is necessary to apply bilateral control in real time. It is well known that in order to increase the task performs, it is important for the human operator to have high tele-presence. Therefore, instead of the original mechanical link between the master-slave system, many bilateral control approaches have been reported. As was pointed in [1], although bilateral control increased tele-presence to some extend, as seen from human operator, the total master--bilateral control block--slave system itself will become a complex dynamic subject, which is difficult for human to operate. In addition, the communication loop time delay will seriously influence the system's stability.

In our bio-mimetic control research, it is found that as seen from brain, our body is also a slave robot, and there also have long time loop delay of $100[\mathrm{~ms}]$ to 200 [ms] from the distal nervous to the high level brain cortex, however we do not have stability problem with respect to such loop delay for a health person. The nerve system at the lower level processed almost all control functions while the higher level of cortex is rarely involved in the motor control. This gives us a hint to increase the autonomous ability of the slave robot.

In our system, we use semi-unilateral control, that is: although the human operator gets tele-presence from interaction with dynamic simulator, the communication between the simulator and the real robot is usually unilateral. The real robot is basically controlled using our novel passive impedance control by considering the time varying impedance center sent from the simulator [2]. The simulator rarely monitors the real environment of the real robot so as to adjust the dynamic model within the simulator.

The last problem then comes from the difference between the model and the real environment, not only dynamics but also the initial geometrical form size and positions. This is our open problem.

For more information, refer to our home page [7].

\section{Conclusions}

Two platforms of immersion type robot experiment systems are developed.

The first system can make the human subject feel naturally as if he/she shares the common environmental space with the virtual robot and performs the physical interactions. This platform will be important for designing, simulating and evaluating of the human interactive robots. At a present stage, development of force display between the subject and the virtual robot is going on, it's our future subject. Moreover, we will measure the joint torque generated while man is operating, using not only motion capture but also multi channels of EMG, force plates and multi channels of accelerometer. It is our future subject for the virtual robot to mimic the skillful and dynamic movement of human subjects.

The second platform can make the human subject feel as if he/she is inside the robot body. Using this system, it's easer to realize the unilateral tele-manipulation with high performance without the stability problem of network communication delay.

\section{References}

[1] Z.W.Luo M.Ito, "Unilateral Control? or Bilateral Control? New Study on Tele-manipulation," Proc. of 1998 Int. Conf. on Mechatronic Technology, pp.509-512, 2003

[2] Y. Kishi, Z.W.Luo, F. Asano, S. Hosoe, "Passive Impedance Control with Time-varying Impedance Center," The $5^{\text {th }}$ IEEE Int. Symposium on Computational Intelligence in Rob. and Auto., 2003.

[3] F. Gravez, B. Mohamed, and F. B. Ouezdou, "Dynamic Simulation of a Humanoid Robot with Four DOFs Torso," Proc. of IEEE Int. Conf. on Rob. and Auto., vol.1, pp.511--516, May 2002.

[4] F. Kanehiro, K. Fujiwara, S. Kajita, K. Yokoi, K. Kaneko, H. Hirukawa, Y. Nakamura, and K. Yamane, "Open Architecture Humanoid Robotics Platform," Proc. of IEEE Int. Conf. on Rob. and Auto., vol.1, pp.24--30, May 2002.

[5] M. Hirose, "CABIN -- A multi-screen display for computer experiments," Proc. of IEEE Int. Conf. on Virtual Sys. and Multi-Media, pp.78--83, Sep. 1997.

[6] T. Mitsuda, S. Kuge, M. Wakabayashi, and S. Kawamura, "Haptic Displays Implemented by Controllable Passive Elements," Proc. of IEEE Int. Conf. on Rob. and Auto., vol.4, pp.4223--4228, 2002.

[7] http://www.bmc.riken.go.jp/ robot/ 